\title{
Una nota sobre la fecha de la muerte de Plinio el Joven*
}

On the date of the Pliny the Younger's death

\section{Marc Mayer i Olivé}

\section{Universitat de Barcelona / Institut d'Estudis Catalans}

mmayer@ub.edu

\begin{abstract}
La fecha tradicionalmente aceptada para la muerte de Plinio el Joven dista mucho de estar suficientemente probada y responde a deducciones hechas a partir del formulario de la titulación imperial de la inscripción que contiene una parte del testamento del escritor. Se suma a ello el aparente silencio de su correspondencia durante la última parte de su gobierno en Bithynia. Estos hechos, que pueden parecer coherentes entre sí, no pueden ser, en nuestra opinión, considerados como una prueba irrefutable, ya que pueden haber sido causados por otros factores, y dicha muerte quizás debería de situarse en un momento algo posterior.
\end{abstract}

Palabras clave: Plinio el Joven; Literatura Latina; Historia de Roma; Cartas de Plinio; Epigrafía.

The date traditionally accepted for the death of the Younger Pliny is far from being sufficiently proven and answers to deductions done from the form of the imperial titulature of the inscription that contains a part of the testament of the writer. There adds the fact of the apparent silence of his correspondence during the last part of his government in Bithynia. These facts that seem to be coherent between them cannot be, in our opinion, considered as an irrefutable test, since they can have be caused by other factors, and Pliny's death probably should be placed in a slightly later moment.

Key Words: Pliny the Younger; Latin Literature; Roman History; Pliny's Letters; Epigraphy.

Uno de los temas que se debaten todavía en la biografía de Plinio el Joven, que fue consul suffectus en el 100 d.C. ${ }^{1}$, es el de la fecha de su muerte, problema que está en íntima relación en opinión de los estudiosos con la no menos controvertida cuestión

* El presente trabajo ha sido realizado en el ámbito del Proyecto de Investigación FFI2011-25113 y del Grup de Recerca Consolidat LITTERA (2009SGR1254).

${ }^{1}$ PIR² P 490, pp. 204-209 (Vidman). Sobre el consulado de Plinio cf. Otto 1919, pp. 1-104, esp. pp. 89-91. 
de la cronología exacta de su gobierno de la provincia de Bithynia ${ }^{2}$. Voluntariamente hemos relegado el status quaestionis reciente a la anotación que acompaña este breve texto, limitándonos en el cuerpo del mismo a referir los puntos principales de la argumentación en que se basa la opinio communis sobre el tema que discutimos; evidentemente las notas recogen los principales trabajos de quienes se han ocupado anteriormente de este problema, así como sus posiciones al respecto, especialmente en lo que concierne a las aportaciones más recientes.

La mayoría de los estudiosos tiende a situar la muerte del escritor y político en torno al 114 d.C., después de su gobierno en Bithynia, donde debió de llegar entre el 110/112 d.C. ${ }^{3}$. Dicho gobierno provincial parece haber coincidido con el de

${ }^{2}$ Cf. Mommsen 1869, pp. 31-139, esp. p. 99 para la muerte del escritor, donde sienta las bases de la opinio communis que hemos recogido para una muerte anterior al 114 d.C., durante su legación o poco después de su regreso de la misma, en pp. 96-98, data la misma en 111 y 112 o en 112 y 113, y su correspondencia se cerraría en la primavera del 112 o del 113 d.C. (= Mommsen 1906, pp. 366-468, esp. pp. 430-433); Magie 1950, p. 605, y esp. p. 1461, nota 23, donde supone que el envío de Plinio a Bithynia respondía ya a la preparación de la posterior campaña de Trajano en Oriente, siguiendo en ello la opinión de Cuntz 1926, pp. 192207; cree también Magie que la estancia de Plinio en la provincia fue inferior a dos años y que aparentemente Gaius Iulius Cornutus Tertullus fue su sucesor en ella. Además, Vidman 1959, pp. 217-225, donde, esp. p. 215, acepta genéricamente las conclusiones de Mommsen al respecto, aunque prefiere la segunda posibilidad, es decir el 111-113 d.C. para el gobierno; el límite ante quem sería el mes de octubre del 113 d.C., cuando Trajano parte de Roma hacia Oriente; Eck 1982, pp. 281-362 y 1983, pp.147-237, esp. pp. 349-350 y nota 275, que lo sitúa en los años 110/111 d.C. y el inicio más probable de su misión el 17 de septiembre del 110 d.C.; cf. además Syme 1988, pp. 478-489, esp. p. 489, donde se inclina por una incorporación de Plinio a su gobierno provincial en el verano del 110 d.C. Una posición tradicional a partir de estos parámetros es la de Durry 1959, pp. VI-VII, que toma como fundamento la inscripción de Como y, evidentemente, la opinión de Mommsen, para concluir: «dès janvier 113 il est de retour en Bithynie. A ce moment la correspondance s'interrompt et il y a lieu de penser que cette interruption est due à la mort de Pline»; o la de Trisoglio 2004, p. 17: «Tuttavia la proposta più persuasiva è che sia giunto in Bitinia il 17 di settembre 111 e ci sia rimasto fino all'inizio del 113», y p. 18: «Con l'inizio del 113 si interrompe la sua corrispondenza ufficiale con Traiano e s'interrompono tutte le notizie di qualsiasi altra fonte: evidentemente Plinio fu colto dalla morte a quella data, mentre era ancora in carica», darle como sucesor a Cornutus Tertullus, su amigo y colega en el consulado del 100 d.C. sería, en opinión de este estudioso, un favor póstumo que el emperador quiso conceder a Plinio. Williams 1990, p. 13, prudentemente afirma al respecto: «However, it must be stressed that these dates are provisional, and it is possible that new discoveries (e.g., about the dates of Calpurnius Macer's term in Lower Moesia) may make necessary to change the dates».

${ }^{3}$ Cf. $R E$ XXI, 1, s.v. «C. Plinius Caecilius Secundus», cols. 439-456 (M. Schuster), esp. col. 440 , donde después de aceptar que «Die Zeit seines Todes ist nicht bekannt», propone: «Wahr- 
Calpurnius Macer, que fue gobernador de Moesia inferior en el $112^{4}$, según parece deducirse de la correspondencia del propio Plinio con Trajano ${ }^{5}$. El silencio de su correspondencia a partir de septiembre del año 112 d.C., ${ }^{6}$ ha sido un factor determinante para suponer su muerte en Bithynia. Su muerte antes del 114 se supone a partir de una inscripción, CIL V 5262=ILS 2927, hoy conservada parcialmente en Milán (fig. 1), que parece corresponder a las termas construidas en Como después

scheinlich hat ihn der Tod ereilt, ehe er aus der Fremde zurück-gekehrt war (um 118)». Sin precisar la fecha pero refiriéndose a la correspondencia con Trajano y las dificultades de Plinio y su gobierno en la reciente monografía de Carlon 2009 se afirma en p. 6: «Pliny did not live to return to Rome from this final service to the emperor». En el mismo sentido se mueve el interesante y escueto capítulo sobre la carrera de Plinio en Birley 2000, pp. 5-17, esp. p. 16: «The only final uncertainty concerns the exact dates of his governorship of Pontus Bithynia (covered by [the letters] x 15-121) and of his death in the province after less than two full years in office», para inclinarse, en p. 17, por el año 112 d.C., como fecha límite para su presencia en la provincia.

${ }^{4}$ Williams 1990 , p. 13, señala que los descubrimientos sobre este personaje cambian la cronología del gobierno de Plinio. La posición tradicional es la mantenida en Sherwin-White 1966, pp. 80-82, que se decanta por 109-111 d.C.; la fecha de la muerte sería en su opinión anterior a la de Trajano y al término de su gobierno provincial. Así indica en p. 82: «That Pliny died before Trajan, and probably before the end of his governorship, is the implication of the long inscription recording his career and his testamentary dispositions, since whatever the missing titles of Trajan may be in it, he is cited as a living emperor. It is unlikely that Pliny would have finished his work in Bithynia or been replaced before he had completed his second year of office, though a successor is not recorded until 113-14», añade a continuación, en p. 84, la opinión de Reynolds, el cual propone como sucesor de Plinio, en el 115-116, a Cornutus Tertullus, aunque la tribunicia potestad del emperador que está presente en la inscripción también podría corresponder al 113-114 d.C.

${ }_{5}$ Plin., Epist. 10, 42 y 62, de Trajano a Plinio; cf. además Epist. 10, 61: Ego interim Cornelio Macro, clarissimo uiro, auctore te scripsi; y también 10. 77, 1, de Plinio a Trajano. Nos servimos de la edición ya mencionada de Trisoglio 2004, en la colección Classici Latini.

${ }^{6}$ Cf. Sherwin-White 1966, pp. 62-65, para la secuencia cronológica de las cartas del libro décimo; para un nuevo resumen cronológico de las cartas desde Bithynia, cf. Williams 1990, p. 18, que data las cartas 104-120 del libro décimo entre el 28 de enero y el 18 de septiembre del 112 d.C. Sobre las características de este último libro de la Correspondencia de Plinio, cf. Ludolph 1997, pp. 49-56. Véase también Vidman 1960, pp. 42-86, para el gobierno de Bithynia. Sobre las cartas en general cf. Aubrion 1989, pp. 304-374, esp. pp. 315-323, para cronología; véase además Prete 1948, esp. el capítulo «Sulla datazione delle lettere», pp. 8496, sobre todo pp. 91-96, donde señala la dificultad de esta datación de la composición para muchas cartas y la existencia de abundantes correcciones antes de hacerlas públicas; Paribeni 1927, pp. 270-271 sobre los retoques hechos por Plinio para publicar sus obras. Cf. tambien Cuntz 1926, p. 197, donde afirma taxativamente que el inicio del mandato provincial de Plinio fue el 17 de septiembre del 111 y que lo mantuvo al menos hasta finales de enero del 113 d.C., fecha de la última carta bien precisada al emperador. 
de su muerte ${ }^{7}$, en la cual Trajano no lleva todavía el apelativo de Optimus, que le fue concedido precisamente el día primero de septiembre de este año ${ }^{8}$. Es ésta la posición de Mommsen que desde 1869 representa el punto firme del que se parte para la datación que podemos considerar consolidada en la mayor parte de los estudios sobre el escritor y su actividad política9.

No obstante, Lagergren publica en 1872 en Upsala una obra sobre la vida y la elocución de Plinio el Joven en la que no podía faltar una referencia a la muerte del autor, que está marcada por una gran prudencia y modestia al aceptar que no se puede determinar ni el momento ni el lugar ${ }^{10}$. Por el contrario Allain publica a principios del siglo XX una obra monumental sobre Plinio el Joven, en la cual se ocupa de la posible fecha de la muerte de este personaje, en una visión teñida de un cierto romanticismo, y la sitúa en un momento del 113 d.C., o algo posterior ${ }^{11}$. La ya clásica monografía

${ }^{7}$ El propio Plinio recuerda en sus cartas estos hechos, cf. Epist. 7, 18, 2 para su legado a la ciudad, y Epist. 1,8, 2 para la biblioteca por él fundada.

${ }^{8}$ Sobre esta inscripción véase la edición de Mommsen 1869, pp. 112-113, y pp. 108-114, para el conjunto de las inscripciones referidas a Plinio (= Mommsen 1906, pp. 444 y 442-446, donde ya está cambiada la restitución en la forma aceptada también por Vidmann); Vidmann 1959, p. 218, que sigue la segunda versión de Mommsen y sitúa missus en el renglón tercero, por lo que puede atribuir a Trajano el título de $p$ (ater) p(atriae) en el renglón siguiente. Una inscripción fragmentaria de Hispellum, CIL XI 5272, contiene una disposición testamentaria de Plinio, como ya había entendido correctamente Bormann, cf. Mommsen 1892, pp. 444445, esta inscripción es añadida en nota en Ges. Schr. IV, p. 444. La titulación del emperador Trajano falta sin embargo en esta última inscripción y se reconstruye tomando como modelo CIL V 5262 de Como, por lo que no se puede, en principio, utilizar como documento probatorio para la cuestión que nos ocupa. La antología de la epigrafía de Trajano, «Inscripciones selectas del reinado de Trajano», recogida en González y Saquete 2003, pp. 349-472, puede ser útil para nuestro objeto. Estos autores datan la inscripción que referimos, CIL V 5262= ILS 2927, por error indicada como CIL III, en el año 113 o 114 d.C., pero la antología misma denota cómo la datación de una parte de las inscripciones se funda en la presencia o no de Optimus para considerarlas posteriores al 114 d.C.

${ }^{9}$ La figura política de Plinio al inicio de su carrera y sus opiniones políticas en relación con la figura de Domiciano han sido analizadas recientemente por Strobel 2003; Beutel 2000, esp. pp. 267-270; además de Soverini 1989, pp. 515-554. Una excelente monografía sobre el tema en general: Trisoglio 1972; puede utilizarse además con provecho el artículo de Cizek 1989, pp. 3-35, esp. pp. 25-32.

${ }^{10}$ Lagergren 1872, p. 5: «Ut autem incertum est quamdiu Plinius prouinciae illi praefuerit, quamquam eum certe plus quam annum hoc munere functum uidemus, ita non magis liquet quando mortuus sit aut ubi, sed cum 46 aetatis annum in uita fuisse, pro certo scimus.»

${ }^{11}$ Allain 1901-1902, esp. vol. III (1902), pp. 222-225, esp. p. 225: «Resté seul après le départ de Calpurnia, Pline dut comme autrefois passer de longues nuits sans sommeil, et "revenir triste et malade, de la chambre toujours vide"; peu après il mourait d'ailleurs a son 


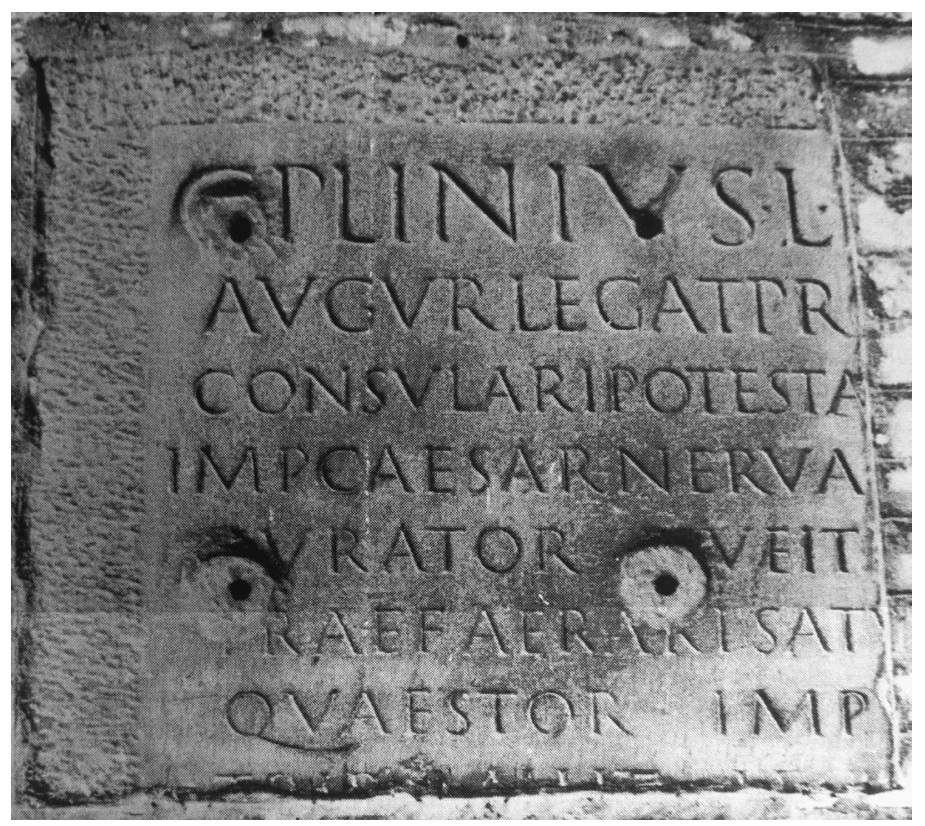

Figura 1. El fragmento de CIL V 5262, procedente de Como y conservado en Milán.

de Guillemin se limita, en cambio, prudentemente a encuadrar la figura de Plinio el Joven entre los intelectuales de su tiempo y en su círculo literario sin llegar a mayores precisiones cronológicas ${ }^{12}$. Una notable excepción viene dada por la contribución de Carcopino, que mantiene que Plinio se retiró de su gobierno provincial para poder

tour, probablement sur la terre lointaine, lamentable exil du fonctionnaire jadis si joyeux, et peut-être sans savoir pu presser sur son coeur "le coeur si fait pour l'aimer"». Sobre la vacilante salud de Plinio el Joven cf. Otto 1919, pp. 58, 68-69, 82, y 93. Sobre Calpurnia: PIR ${ }^{2}$ C 326, p. 78, Berlin, Leipzig 1936 (Groag), cf. Raepsaet-Charlier 1987, núm. 177, pp. 170-171, que cree que fue su segunda esposa.

${ }^{12}$ Guillemin 1929, pero en la biografía, a cargo de M. Durry y de ella misma, incluida en la introducción de su edición de la Correspondencia de Plinio el Joven, Guillemin 1927, p. XXV, se indica explícitamente: «Pline est décédé en charge au début de 113». En el mismo sentido pero con gran prudencia se expresa Zehnacker en su reciente edición: «Le livre X s'arrête brutalement sur cet échange -se refiere a la respuesta de Trajano (10, 120-121)- et on en conclut généralement que Pline a dû mourir peu après, en Bithynie même», a lo que sigue después la discusión sobre el contenido de la inscripción de Como, cf. Zehnaker 2009, pp. XVII-XVIII, con un «Dossier épigraphique» pp. XIX-XXI. 
editar con tranquilidad su obra ${ }^{13}$, con el uso de una abundante documentación que despertó en su momento grandes críticas por su audacia interpretativa ${ }^{14}$.

Nuestra intención en estas breves páginas es analizar la credibilidad de la argumentación aducida para fechar la muerte de Plinio. Señalemos en primer lugar que la inscripción de la que parte la principal prueba menciona efectivamente el nombre del emperador con su consiguiente titulatura en la forma siguiente: [...ab] imp. Caesar Nerua Traiano Aug. German[ico Dacico p. p.], que aunque restituida en parte descarta totalmente el cognomen honoris, Optimus, que se sitúa normalmente entre el nombre del emperador y el apelativo Augustus, lo cual como hemos visto puede tener importantes implicaciones cronológicas para la datación. Las reconstrucciones sucesivas del texto hechas por Mommsen (figs. 2 y 3 ) no difieren substancialmente de la mucho más reciente de Alföldy (fig. 4) para el pasaje al que nos estamos refiriendo, aunque la restitución de la ordinatio sea diversa ${ }^{15}$.

Pero al mismo tiempo podemos notar que las incripciones trajaneas pueden presentar vacilaciones en este aspecto, así CIL VIII 10117=ILS 293, indica la tribunicia potestas y la aclamación imperial correspondientes al 112 y en cambio presenta ya la apelación Optimus que no sería efectiva hasta el 114 d.C. La solución propuesta para esta inscripción por Mommsen y recogida por Dessau, suponía que el texto del epígrafe habría sido concebido en el 112 y habría sido grabado más tarde interpolando

${ }^{13}$ Carcopino 1963, pp. 171-231. esp. p. 179, para su lectura de una parte perdida, línea 13: [item rei p(ublicae) ante tempu]s dedit, de la inscripción de Como, que permitiría datar la inscripción como el agradecimiento a una donación anticipada, y pp. 180-181, para la fecha de redacción de su testamento, anterior a su partida como gobernador; asimismo, pp. 194-196, indica cómo el silencio de su correspondencia, especialmente con Trajano, no parece significativo y utiliza un pasaje del Panegírico $(88,4)$ para demostrar, pp. 217-218, que se recoge en él el título de Optimus que el emperador llevaría más de un decenio después oficialmente, lo que le parece un indicador suficiente para suponer una revisión del Panegírico posterior a 114 d.C. en vistas a su publicación. Evidentemente nuestro razonamiento no entrará en la discusión de las afirmaciones de Carcopino, ni entrará en las vías por las que discurre su indudablemente brillante argumentación, aunque los resultados pueden parecer coincidentes en alguno de los aspectos y en la constatación de las ambigüedades e imprecisiones de la tradición aceptada que dan lugar a estas breves páginas. Se nota especialmente esta discrepancia en la no consideración de sus conjeturas para la inscripción de Como y en el tratamiento de los documentos epigráficos.

${ }^{14}$ Cf. la crítica de Durry, 1964-1965, pp. 5-8 y la de Hanslik 1964, cols. 1-16. Además Aubrion 1989, p. 310. Por el contrario Bardon 1964, p. 1001, manifiesta que la hipótesis interpretativa de Carcopino para la inscripción de Como vale cuanto la de Mommsen.

${ }^{15}$ Cf. la importante contribución de Alföldy 1999a, esp. pp. 28-31, y Alföldy 1999b esp. pp. 227-229 y 243, en un más reciente reexamen del texto de CIL V 5262; además Eck 2001, esp. pp. 226-229 y esp. p. 229, donde acoge las correcciones de Alföldy. De forma general para la liberalitas de Plinio hacia su ciudad natal Manuwald 2003. 


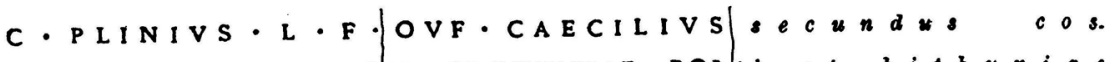

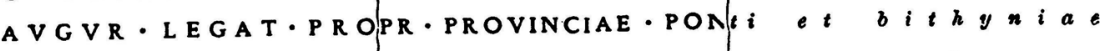
CONSVLARI - POTESTA/t $\cdot \mathrm{IN} \cdot \mathrm{EAM} \cdot \mathrm{PROVINCIAM} \cdot \mathrm{E} x$ s. c. a $b$ IMP - CAESAR - NERVA TRAIANO - AVG - GERMANico dacico missus 5 CVRATOR.ALVEI-T IbERIS - ET - RIPARVM - Et c l o a car. urb. PRAEF - AERARI S A T VrNI - PRAEF - AERARI - MIL $i t . p r . t r i b . p l$ QVAESTOR. IMP.|SEVIR - EQVITVM $\quad r 0 m a n 0 r u m$

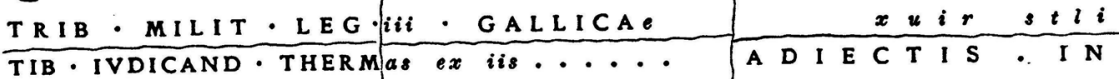
OR NATVM - IIS - CCC..... et eo ampLIVS I IN T T TELAm

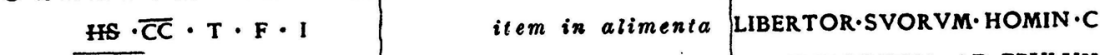
HS - XVIII LXVI BCLXVI REIp. legauit, quorum incREMENT·POSTEA·AD·EPVLVM

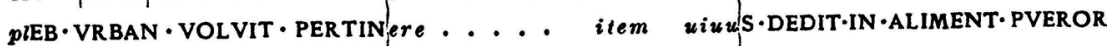

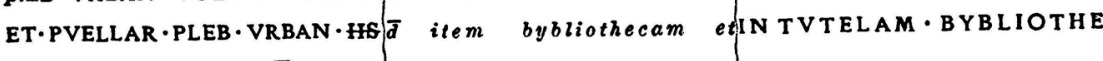
CAE $\cdot$ HS $\cdot \bar{C}$

Figura 2. CIL V 5262=ILS 2927, según la reconstrucción de Mommsen 1869, p. 112.

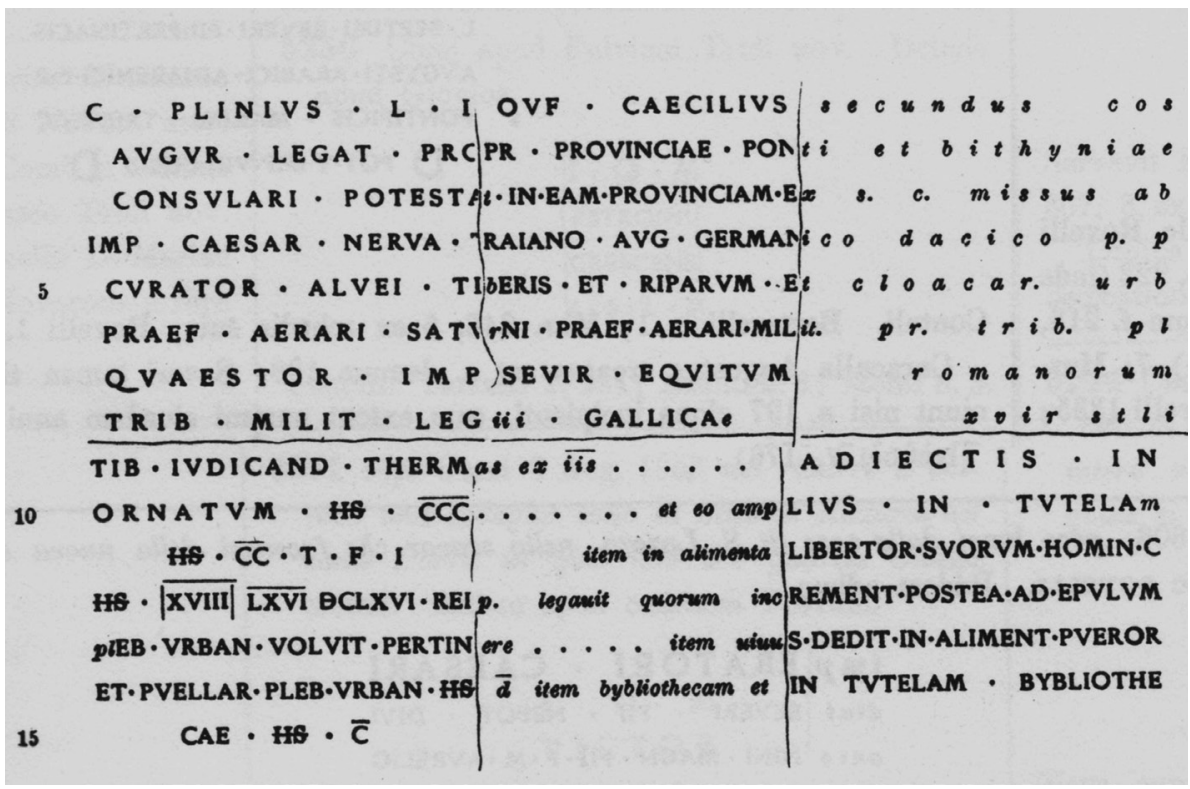

Figura 3. Reconstrucción de Mommsen publicada en CIL V 5262, p. 568. 


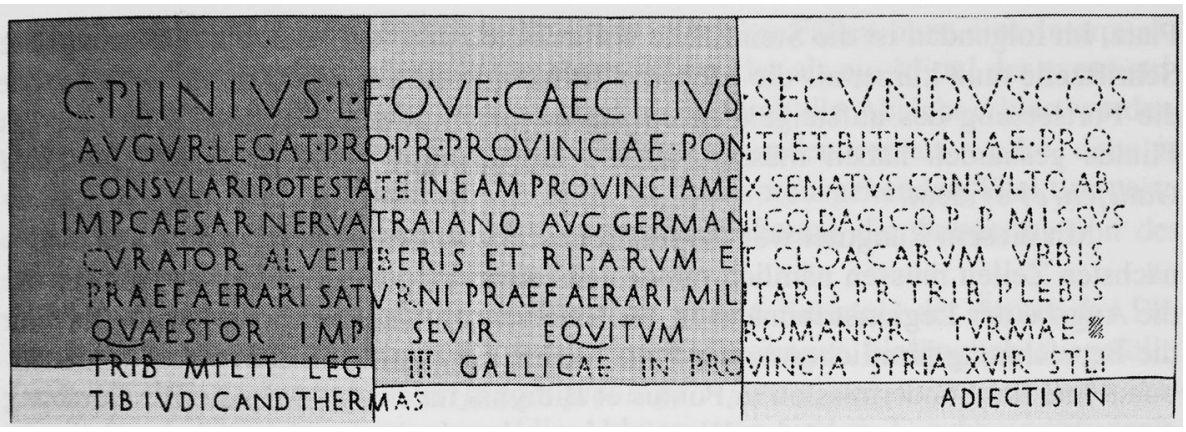

Fig. 4. Reconstrucción de Alföldy publicada en Alföldy 1999a, p. 31.

en él el nuevo apelativo ${ }^{16}$, explicación tan posible como artificiosa. Por otra parte $C I L$ III 6359=ILS 2665 de Risinium se data en el 116 d.C. y lleva el agnomen Optimus a continuación de Parthicus $^{17}$ lo cual constituye aparentemente una anomalía notable. $A E$ 1933, 28 de Pérgamo, posterior al 117 d.C., se refiere al emperador, ya difunto, únicamente como Sebastos, en una inscripción en la que se hace constar que el cónsul Gayo Julio Cuadrato Baso acompañó, como legado de Adriano, el cuerpo de Trajano desde Selinunte y que documenta un uso claro de lo que después será la indicación del diuus Traianus como Parthicus, su principal título de victoria, habitual en la epigrafía posterior, que parece no conservar en ningún caso el título de Optimus. ${ }^{18}$ Una cuestión distinta es la de los diplomas militares de su momento, que deben insistir forzosamente en la titulatura oficial.

Creemos por consiguiente que la ausencia del apelativo Optimus en la inscripción de Como podría no resultar tan trascendente como hasta ahora se ha supuesto, ya que pudo incluso estar desplazado en la zona perdida del epígrafe o simplemente no estar presente, sin que ello pueda considerarse un indicio cronológico determinante definitivo.

Pensemos además que el testamento de Plinio pudo ser redactado bastante antes de su muerte y, conociendo su carácter de administrador minucioso, debió prever hasta los últimos detalles entre los que se pudo contar la inscripción que nos ocupa, que presenta bastantes trazas de originalidad en la mención de sus propios cargos ${ }^{19}$, hecho que resulta suficiente para llegar a creer que pudo haber salido en gran parte

${ }^{16}$ Anotación a ILS 293, ILS, vol. I, Berlin $1954^{2}$, p. 77.

17 González y Saquete 2003, núm. 148, pp. 458-459.

${ }_{18}$ González y Saquete 2003, pp. 458-465.

19 Vidman 1959, p. 251, la cree seguramente del 113 d.C. por no mencionar los títulos de Optimus y Parthicus. Sobre los cargos de Plinio para su gobierno y su originalidad, causada en parte por el hecho de que una provincia senatorial pasaba temporalmente al control imperial, 
de la mano del propio Plinio en una voluntad de fijar exactamente su memoria. Evidentemente en el momento que Plinio fue missus a la provincia de Bithynia, el emperador no llevaba todavía apelativo Optimus por lo que en un texto, que pudo pretender fijar la memoria histórica veraz de un cursus, no resultaba necesario usarlo y hubiera resultado además anacrónico. La posibilidad de un error en la titulatura del emperador puede considerarse descartable en una inscripción tan cuidada y minuciosa en sus detalles. Estas constataciones pueden resultar válidas si pretendemos que la muerte de Plinio fue posterior al 114 d.C. y que la inscripción pudo ser redactada por él mismo. Si situamos su muerte, como acostumbra a hacerse, en un momento anterior al primero de septiembre de dicho año, la salvedad que proponemos resulta inútil, pero no excluye que el propio Plinio pueda haber sido el autor del epígrafe en función de sus características.

Nos parece, no obstante, que resulta claro que del texto de la inscripción de Como no puede inferirse automáticamente que sea anterior al primero de septiembre del 114 d.C. Emerge de ello una única constatación segura: fue redactada antes de la muerte de Trajano, ya que la indicación de la misma, mediante la forma diuus, no hubiera podido pasar por alto al redactor o al ejecutor, que seguramente habría acortado también la titulatura imperial que está presente en el epígrafe.

El hecho concomitante que parece haber desencadenado la datación, es decir el cese de su correspondencia, nos parece, al menos desde un punto de vista literario, irrelevante y sin consecuencia cronológica e histórica cierta ${ }^{20}$. Sabemos de la dedicación de Plinio a cuidar su propia imagen a través de la correspondencia, y tenemos sobradas pruebas de la reelaboración de sus cartas antes de hacerlas públicas ${ }^{21}$. Evidentemente la colección transmitida por la tradición no corresponde a la totalidad de dicha correspondencia sino a una reelaboración y selección determinadas por su propio autor; de aquí se infiere que el término final de la misma no resulte elemento dirimente. Pudo quedar una parte posterior en preparación, o pudo simplemente Plinio decidir que ponía punto final a la serie que quiso hacer pública con algunas de las cartas escritas durante su gobierno en Bithynia.

El relativo malestar de Plinio en la parte considerada final de su correspondencia resulta evidente y sabemos que aproximadamente en aquel momento abandonó su gobierno provincial presumiblemente para volver a Roma. Suponer que su muerte

véase el claro resumen de Durry 1959, pp. VII y X, donde insiste en que CIL V 5262, de Como, refleja exactamente esta situación, que había notado Mommsen, cf. nota 2.

${ }^{20}$ Recordemos que, como señala correctamente Vidman 1959, p. 23, se produce una interrupción importante, aproximadamente de diez años, de la correspondencia entre Plinio y Trajano comprendida entre el 102 d.C. y el momento del inicio de su gobierno provincial.

${ }^{21}$ Véanse las notas 6 y 13; además Aubrion 1989, p. 315, para las posiciones sobre la publicación de las obras, y Soverini 1989, pp. 538-539, para la ampliación del Panegírico. 
coincide con este hecho no se deduce de ninguna información, ni la propia correspondencia nos da motivo real para hacerlo. Se trata claramente de un argumento ex silentio. El intentar deducir además que su muerte se produciría en Bithynia no es más que sumar una conjetura a otra. Podemos elevar a categoría lo que ya constató en su momento Syme para este tipo de argumentaciones: «The validity of arguments of silence varies enormously. Silence can sometimes be valuable, as when it helps to circumscribe the "circle of Pliny" by invoking categories of persons who receive no letters. In this instance, doubts have been conceived $»^{22}$.

A la vista de la debilidad de ambos argumentos, que parecen darse mutua fuerza sólo aparentemente, no se puede aceptar sin más que la muerte de Plinio debió de producirse en un momento anterior al primero de septiembre del año 114 d.C., aunque tuvo lugar, evidentemente, con posterioridad a la fecha aproximada de su última carta a Trajano; mucho menos todavía se puede afirmar que dicha muerte tuviera lugar fuera de Italia.

Las explicaciones alternativas podrían ser muchas. No ha dejado de proponerse el cansancio y la desazón de Plinio a su regreso a Roma y su retirada a la vida privada. Pudo poner un punto final brusco a su gobierno una posible pérdida del favor imperial por un motivo que nos es por ahora desconocido. Existe también la posibilidad de que sus enemigos le hubieran provocado un proceso, incluso como venganza de los que él mismo había ganado contra otros gobernadores de provincia ${ }^{23}$. La retahíla de conjeturas podría ser larga, pero entrar en su consideración sería encaminarnos de nuevo por un camino de encabalgamientos de hipótesis que es precisamente lo que hemos querido, en la medida de lo posible, evitar y poner en evidencia en estas páginas. Un hecho resulta evidente: la muerte de Plinio el Joven debió de producirse probablemente en un momento anterior a la de Trajano en agosto del año 117 d.C. ${ }^{24}$, pero no forzosamente al término de su gobierno en Bythinia, ni siquiera inmediatamente después, como se ha tendido a considerar en la «communis opinio», cuyos razonamientos, como hemos visto, distan mucho de poder ser considerados como irrebatibles y deben ser, como mínimo, tratados con reserva.

\section{BIBLIOGRAFÍA}

Alföldy, G. 1999a: «Die Inschriften des Jüngeren Plinius und seine Mission in Pontus et Bithynia», Acta Ant. Hung. 39, pp. 21-44.

22 Syme 1989, p. 489.

${ }^{23}$ Así el de Mario Prisco gobernador de África, o bien la acción contra Cecilio Clásico gobernador de la Bética, cf. Plin., Epist, 3, 9.

${ }^{24}$ Cf. RE Supplementband X, Stuttgart 1965, s. v. «M. Ulpius Traianus», cols. 1035-1102 (Hanslik)), esp. cols. 1101-1102. 
Alföldy, G. 1999b, Städte Eliten und Gesellschaft in der Gallia Cisalpina. Epigraphischhistorische Untersuchungen (HABES 30), Stuttgart, pp. 221-244.

Allain, E. 1901-1902: Pline le Jeune et ses héritiers, 4 vols. París.

Aubrion, E. 1989: «La "Correspondance" de Pline le Jeune: Problèmes et orientations actuelles de la recherche», en $A N R W$ II, 33, 1, Berlín - Nueva York, pp. 304-374.

Bardon, H. 1964: recensión a Carcopino 1963, Revue Belge de Philologie et d'Histoire 42, pp. 1001-1003.

Beutel, F. 2000: Vergangenheit als Politik. Neue Aspekte im Werk des jüngeren Plinius, Frankfurt a.M. (Studien zur klassischen Philologie 121).

Birley, A. R. 2000: Onomasticon to the Younger Pliny, Múnich, Leipzig.

Carcopino, J. 1963b: Rencontres de l'histoire et de la littérature romaines, Paris («Les surprises du testament de Pline le Jeune», pp. 171-231).

Carlon, J. M. 2009: Pliny's Women. Constructing Virtue and Creating Identity in the Roman World, Cambridge.

Cizek, E. 1989: «La littérature et les cercles culturels et politiques à l'époque de Trajan», en ANRW II 33, 1, pp. 3-35.

Cuntz, O. 1926: «Zum Briefwechsel des Plinius mit Trajan», Hermes 61, pp. 192-207.

Durry, M. 1959²: «Introduction», en Pline le Jeune, Lettres. Libre X. Panégyrique de Trajan, París (Collection des Universités de France).

Durry, M. 1964-1965: «Travaux récents sur Pline le Jeune, Journées d'études». Conf. de la Soc. d'Ét. Lat de Bruxelles 37, pp. 5-8.

Eck, W. 1982: «Jahres- und Provinzialfasten der senatorischen Statthalter von 69/70 bis 138», Chiron 12, pp. 281-362 y 13, pp.147-237.

Eck, W. 2001: «Die große Pliniusinschrift aus Comum: Funktion und Monument», en Bertinelli, G. A. y Donati, A. eds., Varia Epigraphica. Atti del Coloquio Internazionale di Epigrafia, Bertinoro 8-10 giugno 2000, Faenza (Epigrafia e antichità, 17), pp. 225-235.

González, J. y Saquete, C. (coords.) 2003: Marco Ulpio Trajano emperador de Roma. Documentos y fuentes para el estudio de su reinado, Sevilla.

Guillemin, A. M. 1927: Pline le Jeune Lettres, Tome I Livres I-III, París.

Guillemin, A. M. 1929: Pline et la vie littéraire de son temps, París.

Hanslik, R. 1964: «Plinius der Jüngere. Forschungsbericht», Anzeiger für die Altertumswissenschaften, 17, cols. 1-16.

Lagergren, J. P. 1872: De vita et elocutione C. Plinii Caecilii Secundi, Upsala.

Ludolph, M. 1997: Epistolographie und Selbstdarstellung. Untersuchungen zu den «Paradebriefen» Plinius des Jüngeren, Tubinga (Classica Monacensia,17).

Magie, D. 1950: Roman Rule in Asia Minor to the End of the Third Century after Christ, Princeton.

Manuwald, G. 2003: «Eine "Schule" für Novum Comum (Epist. 4, 13) Aspekte der liberalitas des Plinius» en Castagna, L. y Lefèvre, E. (eds.), Plinius der Jüngere und seine Zeit, Múnich-Leipzig, pp. 203-217.

Mommsen, Th. 1869: «Zur Lebensgeschichte des jüngeren Plinius», Hermes 3, pp. 31-139.

Mommsen, Th. 1892: «XLV. Titulus Plinii Hispellas», en EE VII, Berlín.

Mommsen, Th. 1906: Gesammelte Schriften, vol. IV, Berlín. 
Otto, W. 1919: Zur Lebensgeschichte des jüngeren Plinius, Múnich (Sitzungsberichte der Bayerische Akademie der Wissenschaften. Philos.- philol. und hist. Klasse, Jahrgang 10. Abhandlung).

Paribeni, R. 1927: Optimus princeps. Saggio sulla storia e sui tempi dell'imperatore Traiano, vol. II, Mesina.

Prete, S. 1948: Saggi Pliniani, Bolonia (Studi pubblicati dall'Istituto di Filologia Classica, III).

Raepsaet-Charlier, M. Th. 1987: Prosopographie des femmes de l'ordre sénatorial (I ${ }^{e r}-I^{e}$ s.), Lovaina (Académie Royale de la Belgique. Classe des Lettres, Fonds René Draguet, IV).

Sherwin-White, A. N. 1966: The Letters of Pliny. A Historical and Social Commentary, Oxford.

Soverini, P. 1989: «Impero e imperatori nell'opera di Plinio il Giovane: Aspetti e problemi del rapporto con Domiziano e Traiano», en $A N R W$ II 33, 1, Berlín, Nueva York, pp. 515-554.

Strobel, K. 2003: «Plinius und Domitian: Der willige Helfer eines Unrechtssystems? Zur Problematik historischer Aussagen in den Werken des jüngeren Plinius», en Castagna, L. y Lefèvre, E. (eds.), Plinius der Jüngere und seine Zeit, Múnich-Leipzig, pp. 303-314.

Syme, R. 1988: «The Dating of Pliny's Latest Letters», en Id., Roman Papers, vol. V, Oxford (ed. por A. R. Birley), pp. 478-489 (publicado por primera vez en 1985, CQ XXXV, pp. 176-185).

Trisoglio, F. 1972: La personalità di Plinio il Giovane nei suoi rapporti con la politica, la società e la letteratura, Turín (Memorie dell' Accademia delle Scienze di Torino, Classe di Scienze Morali, Storiche e Filologiche, ser. 4, núm. 25).

Trisoglio, F. 2004: Opere di Plinio Cecilio Secondo, Turín (reimpr. de la ed. de 1973) (Classici Latini).

Vidman, L. 1959: «Die Mission Plinius’ des Jüngeren in Bithynien», Klio 37, pp. 217-225.

Vidman, L. 1960: Étude sur la correspondance de Pline le jeune avec Trajan, Praga (Rozpravy Českoslovenenské Akademie Vèd, 70, 14).

Williams, W. 1990: Pliny. Correspondence with Trajan from Bithynia (Epistles X), Warminster. Zehnacker, H. 2009: Pline Le Jeune. Lettres Livre I-III, París (Collection des Universités de France).

Fecha de recepción: 14/01/2013

Fecha de aceptación: 30/08/2013

Fecha de recepción de versión definitiva: 26/11/2013 